\title{
Anisotropic zero-resistance onset in organic superconductors
}

\author{
Vladislav D. Kochev, ${ }^{1}$ Kaushal K. Kesharpu, ${ }^{1}$ and Pavel D. Grigoriev ${ }^{2,1,3, *}$ \\ ${ }^{1}$ National University of Science and Technology MISiS, 119049, Moscow, Russia \\ ${ }^{2}$ L. D. Landau Institute for Theoretical Physics, 142432, Chernogolovka, Russia \\ ${ }^{3}$ P. N. Lebedev Physical Institute, RAS, 119991, Moscow, Russia
}

\begin{abstract}
We study the coexistence of superconductivity (SC) and density-wave state and reconcile various puzzling experimental data in organic superconductors (TMTSF) ${ }_{2} \mathrm{PF}_{6}$ and (TMTSF) $)_{2} \mathrm{ClO}_{4}$. The anisotropic resistance drop above $T_{c}$ is qualitatively described by nascent isolated SC islands within a bulk analytical model. However, the observed anisotropic SC onset is explained only when the finite size and flat needle shape of samples is considered. Our results pave a way to estimate the volume fraction and the typical size of SC islands in far from the sample surface, and apply to many inhomogeneous superconductors, including high- $T_{C}$ cuprate or Fe-based ones.
\end{abstract}

\section{INTRODUCTION}

The interplay between various types of electronic ordering is a subject of extensive research in condensed matter physics. It is crucial for understanding the electronic properties of various strongly correlated electron systems. The coexistence of charge- or spin-density wave (CDW/SDW) and superconductivity (SC) is very common [1- -3$]$ and especially important for high- $T_{c}$ superconductors, both cuprate [4-6] and iron-based [7, 8], for transition metal dichalcogenides [9, 10] and tetrachalcogenides [3], for organic superconductors [11-22]. In these materials the density wave (DW) is suppressed by some external parameter, such as pressure or doping. The SC transition temperature $T_{c}$ is, usually, the highest in the coexistence region near the quantum critical point where DW disappears. The upper critical field $H_{c 2}$ is often several times higher in the coexistence region than in a pure SC phase [13, 22], suggesting possible applications of SC/DW coexistence.

The microscopic structure of SC and DW coexistence is important for understanding the DW influence on SC properties and $\mathrm{SC}$ transition temperature $T_{c}$. The DW and SC phase separation may happen in the momentum or coordinate space. The first scenario assumes a spatially uniform structure, when the Fermi surface is partially gapped by DW and the ungapped parts give SC [3, 23]. The second scenario means that SC and DW phases are spatially separated on a microscopic or macroscopic scale, depending on the ratio of SC domain size $d$ and the SC coherence length $\xi_{S C}$. An example of microscopic SC domains with size $d<\xi_{S C}$ is the soliton DW structure, where SC emerges in the soliton walls [24-28]. The SC upper critical field $H_{c 2}$ may theoretically increase several times in both coexistence scenarios [23, 26].

It is yet unknown or debated how SC and DW coexist even in the relatively weakly correlated organic superconductors, such as (TMTSF) $)_{2} \mathrm{PF}_{6}$ [14-17], (TMTSF) ${ }_{2} \mathrm{ClO}_{4}[20,21]$ or $\alpha$ $(\mathrm{BEDT}-\mathrm{TTF})_{2} \mathrm{KHg}(\mathrm{SCN})_{4}$ [22]. Among these materials the most extensive and detailed experimental data are available for (TMTSF) ${ }_{2} \mathrm{PF}_{6}$ [11-19]. This compound attracts special attention because superconductivity there appears on a spindensity wave background, which violates the conservation of

* grigorev@itp.ac.ru

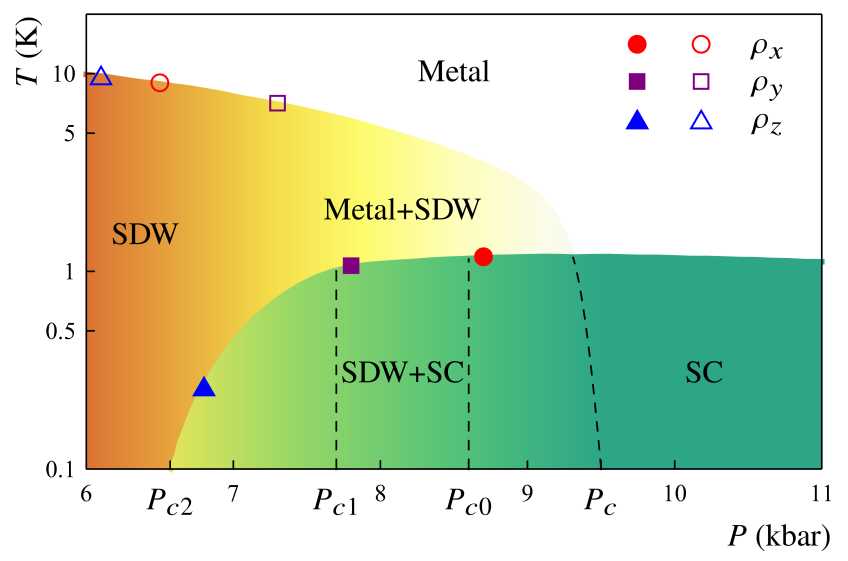

FIG. 1. Pressure-temperature phase diagram of (TMTSF $)_{2} \mathrm{PF}_{6}$ recreated from resistivity data in Ref. [15]. Filled (blank) symbols show the transition towards SC (SDW) phase. The intensity of green (orange) color shows the SC (SDW) volume fraction coexisting with the SDW (metal) phase.

electron spin and, in the case of a microscopic SDW/SC coexistence, favors [23, 28] the unconventional spin-triplet SC. The latter is supported by the observed high in-plane upper critical field [18], exceeding several times the expected paramagnetic limit, and by the NMR Knight shift measurements [19]. However, an indisputable experimental confirmation of a triplet $\mathrm{SC}$ in (TMTSF) ${ }_{2} \mathrm{PF}_{6}$ is still missing.

At ambient pressure (TMTSF) ${ }_{2} \mathrm{PF}_{6}$ undergoes a transition from metallic to SDW insulating state at temperature $T_{C S D W} \approx 10 \mathrm{~K}$. The SDW transition temperature decreases with the raise of pressure [11-16], and SDW becomes finally suppressed at $P_{c} \approx 9.5 \mathrm{kbar}[11,-16,29]$, as shown in Fig. 1. At pressure exceeding $P_{c 2}<P_{c}$ superconductivity emerges at $T<T_{c} \approx 1.1 \mathrm{~K}$. The temperature hysteresis observed [14] in the SDW/metal or SDW/SC coexistence region in (TMTSF) ${ }_{2} \mathrm{PF}_{6}$ suggests a spatial rather than momentum separation of the metal/SC and SDW phases. However, the origin, size and shape of SC/metal domains in SDW phase remains unknown and debated [15, 16], because various observations seem to contradict each other in a framework of any SC/SDW coexistence model. For example, the strong increase in $H_{c 2}$, both perpendicular [13, 22] and parallel [18] to con- 
ducting layers, and the "spin-triplet" SC properties [18, 19] suggest a microscopic SDW/SC coexistence, e.g. the domainwall scenario [15, 24-28]. On the other hand, the angular magnetoresistance oscillations (AMRO) observed in the pressure interval of SC/SDW coexistence in (TMTSF) ${ }_{2} \mathrm{PF}_{6}$ can be explained only by assuming a macroscopic spatial phase separation with SC domain width $d>1 \mu \mathrm{m}$ [16].

The most puzzling feature of SDW/SC coexistence in (TMTSF) ${ }_{2} \mathrm{PF}_{6}$, unexplained in any scenario, is the anisotropic SC onset [15, 16]: with the increase in pressure at $P_{c 2} \approx 6.7$ kbar the SC transition and the zero resistance is first observed only along the least-conducting interlayer $z$-direction, then at $P_{c 1} \approx 7.8 \mathrm{kbar}$ along $z$ - and $y$-directions, and only at $P_{c 0} \approx 8.6 \mathrm{kbar}$ in all directions, including the most conducting $x$-direction. This is opposite to a weak intrinsic interlayer Josephson coupling, typical in high- $T_{c}$ superconductors [30]. Other organic metals manifest similar anisotropic SC onset [20]. Note that the observed [15, 16] anisotropic zeroresistance $T_{c}$ contradicts the general rule that the percolation threshold in large heterogeneous media must be isotropic [31], provided the high-conducting inclusions are not thin filaments [15] connecting opposite edges of a sample. However, such a filament scenario cannot be substantiated microscopically in (TMTSF) ${ }_{2} \mathrm{PF}_{6}$ and seems to be absent in the metal/SDW coexistence region at $T>T_{c}$. Below we resolve this paradox and reconcile relevant experimental data on SC onset in (TMTSF $)_{2} \mathrm{PF}_{6}$. The proposed model and the results obtained are applicable to many other superconductors and can be used to estimate the volume fraction and the size of SC domains.

\section{MODEL AND CALCULATIONS}

\section{A. Resistivity anisotropy above $T_{C}$ in large samples}

A possible clue to explain the observed SC anisotropy without invoking SC filaments may come from a similar effects in iron selinide FeSe, where the resistivity drop $\Delta \rho$ above $T_{c}$ was also observed to be very anisotropic, being much greater along the least conducting interlayer direction [32, 33]. Its superconducting origin was confirmed by the simultaneous measurements of a diamagnetic response and of the critical current [32]. This SC anisotropy was explained within a model of a heterogeneous SC onset in the form of isolated $\mathrm{SC}$ islands [32, 33]. This effect originates from a strong conductivity anisotropy $\eta_{z}=\sigma_{z z}^{0} / \sigma_{x x}^{0} \ll 1$ of the parent non-SC material [34] and takes place if SC islands are spheres [32] or even flattened spheroids [33], opposite to filaments along $z$-axis. Isolated spherical SC islands increase conductivity in all directions similarly, but their relative effect $\Delta \sigma_{i} / \sigma_{i i}^{0}$ for the interlayer current is $\sim 1 / \eta_{z} \gg 1$ times greater than for the in-plane current. An analytical description of this effect in fully anisotropic compounds, i.e. with $\eta_{y}=\sigma_{y y}^{0} / \sigma_{x x}^{0}<1$ and elliptic SC inclusions with main semiaxes $a_{i}$, can be obtained using the Maxwell-Garnett approximation (MGA), valid in the limit of small volume fraction $\phi \ll 1$ of SC phase, or the self-consistent approximation (SCA), describing specific spatial distributions of the second phase [35]. These models
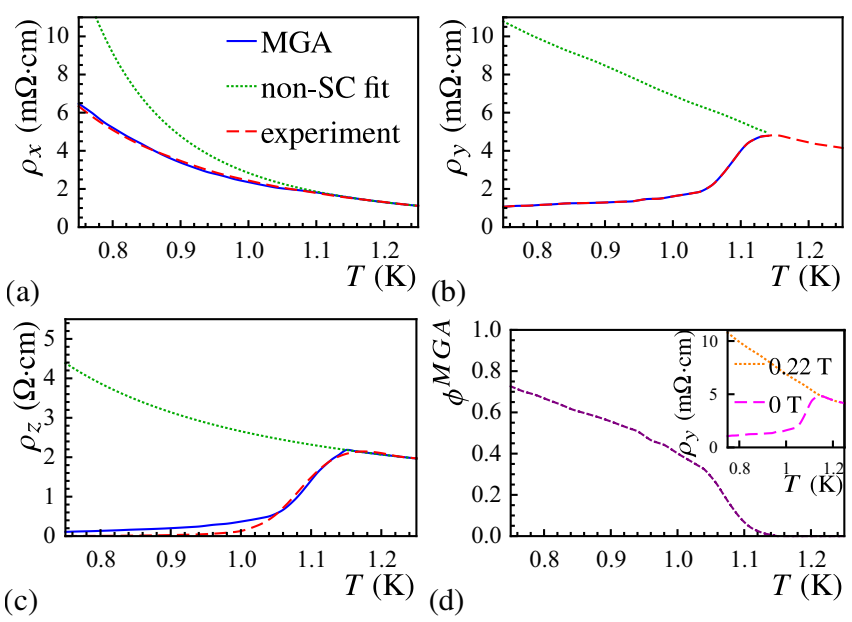

FIG. 2. Temperature dependence of resistivity $\rho$ along (a) $x$, (b) $y$ and (c) $z$ axes. Used experimental data for (TMTSF) ${ }_{2} \mathrm{PF}_{6}$ at $P=8.3$ kbar were extracted from Fig. 4a of Ref. [15] Plotted lines correspond to: calculation in MGA using Eq. (1) (solid blue); extrapolated resistivity before the $\mathrm{SC}$ onset (dotted green); experiment (dashed red). (d) Temperature dependence of SC volume ratio calculated using Eq. (4) and experimental $\rho_{y}$ in magnetic field (inset) at $B=$ $0.22 \mathrm{~T}$ and $B=0 \mathrm{~T}$.

were derived in the bulk limit of infinitely large samples [35]. In MGA the resistivity $\rho_{i}=1 / \sigma_{i i}$ along the axis $i \in\{x, y, z\}$ is given by [36]:

$$
\rho_{i}^{M G A}=\rho_{i}^{0}\left[\frac{A_{i}^{*}(1-\phi)}{A_{i}^{*}+\left(1-A_{i}^{*}\right) \phi}\right],
$$

while in SCA we obtain (details of derivation presented in Appendix A]:

$$
\rho_{i}^{S C A}=\rho_{i}^{0}\left(1-\phi / A_{i}^{*}\right),
$$

where the diagonal components of depolarization tensor are given by Eq. (17.25) of Ref. 35 :

$$
A_{i}^{*}=\frac{1}{2} \prod_{n=1}^{3} a_{n}^{*} \int_{0}^{\infty} \mathrm{d} t\left[\left(t+a_{i}^{* 2}\right) \sqrt{\prod_{n=1}^{3}\left(t+a_{n}^{* 2}\right)}\right]^{-1},
$$

where $a_{i}^{*}=a_{i} / \sqrt{\eta_{i}}, \eta_{i}=\sigma_{i i} / \sigma_{x x}$.

Unfortunately, the SCA gives a qualitatively incorrect result in the limit of strong anisotropy $\eta_{i} \ll 1$ and strong conductivity contrast [35], when the conductivity of two phases differ too much, as in our case of SC inclusions: $\sigma^{S C} / \sigma^{0}=\infty$. This is illustrated by our numerical calculations in $2 \mathrm{D}$ case shown in Fig. 5 (see Appendix B). From Eq. (1) one can also solve an inverse problem to express the volume fraction $\phi$ through the conductivity with and without SC inclusions:

$$
\phi^{M G A}=\frac{A_{y}\left(\sigma_{y y}-\sigma_{y y}^{0}\right)}{\sigma_{y y}^{0}+A_{y}\left(\sigma_{y y}-\sigma_{y y}^{0}\right)} .
$$

We apply Eqs. (1)-(4) to fit the observed resistivity anisotropy $\rho_{i}(T)$ in (TMTSF) $)_{2} \mathrm{PF}_{6}\left[15\right.$, 16] at $T>T_{c}$ (see 
Fig. 22. The required temperature dependence $\phi(T)$ is extracted using Eq. (4) from the resistivity data [15] without and with magnetic field destroying SC (see Fig. 2d). From Fig. 2 one sees that the observed very anisotropic temperature dependence of resistivity $\rho_{i}(T)$ is qualitatively described by isolated SC islands within MGA. The effect of SC inclusions on resistivity in MGA is clearly seen from the difference between the solid blue and dotted green curves in Fig. 2, showing $\rho_{i}(T)$ with and without SC islands. However, the MGA cannot explain the anisotropic zero-resistance onset observed in $(\mathrm{TMTSF})_{2} \mathrm{PF}_{6}\left[15\right.$, 16] and $(\mathrm{TMTSF})_{2} \mathrm{ClO}_{4}$ [20], i.e. the anisotropy of $\mathrm{SC}$ transition temperature $T_{c}$ where the observed resistivity drops by several orders of magnitude. Moreover, such a $T_{c}$ anisotropy seems to contradict the percolation theory [31].

\section{B. Finite-size effects and zero resistance onset}

To resolve this puzzle we note that the percolation threshold is isotropic only in infinite heterogeneous media [31], i.e. when the sample dimensions are much larger than the size $d$ of SC islands. Usually, the single crystals of organic metals are flat whiskers elongated in the most conducting $x$-direction with a tiny thickness along the interlayer $z$-axis. The (TMTSF) ${ }_{2} \mathrm{PF}_{6}$ samples in the experiments of Refs. 14 and 15 were $3 \times$ $0.2 \times 0.1 \mathrm{~mm}^{3}$. The typical dimensions of (TMTSF) ${ }_{2} \mathrm{ClO}_{4}$ single crystals are similar: $3 \times 0.1 \times 0.03 \mathrm{~mm}^{3}$ in Ref. 20 , or $2.4 \times 0.7 \times 0.1 \mathrm{~mm}^{3}$ in Ref. 21. The observation of AMRO and FISDW in (TMTSF) ${ }_{2} \mathrm{PF}_{6}$ at field $B \approx 2 \mathrm{~T}$ restricts the minimal size $d_{\min }$ of SC islands to $d_{\min }>1 \mu \mathrm{m}$ [16]. On the other hand, the observed [13, 22] increase in $H_{c 2}$ restricts the maximal SC size to $d_{\max }<\lambda$, where the penetration depth $\lambda(T=0.19 \mathrm{~K}) \approx 40 \mu \mathrm{m}$ in (TMTSF $)_{2} \mathrm{ClO}_{4}$ [37], and a close $\lambda$ is expected in (TMTSF) ${ }_{2} \mathrm{PF}_{6}$ [38]. Similar $H_{c 2}$ enhancement and AMRO were also observed in (TMTSF) ${ }_{2} \mathrm{ClO}_{4}$ [20, 39]. These experimental data suggest that the typical size $d$ of SC islands in (TMTSF) ${ }_{2} \mathrm{PF}_{6}$ and (TMTSF) ${ }_{2} \mathrm{ClO}_{4}$ gets into the interval $1 \mu \mathrm{m}<d \lesssim 40 \mu \mathrm{m}$, being comparable to the sample thickness $L_{z} \sim 100 \mu \mathrm{m}$. Thus we need to analyze the effect of finite sample size.

For this end we calculated percolation thresholds $\phi^{c}$ numerically for randomly distributed spherical SC inclusions of various diameter $d$ in a sample of dimensions $3 \times 0.2 \times 0.1$ $\mathrm{mm}^{3}$, as in the experiment [14, 15]. For $d>10 \mu \mathrm{m} \phi^{c}$ strongly depends on the distribution pattern of SC islands, hence, the percolation probabilities $p\left(\phi^{c}\right)$ in Fig. 3 obtained by averaging over the large number of distribution patterns (see Appendix C for details of calculations). In Fig. 3 we see that $p$ is the largest along the shortest sample dimension in all cases. With the increase in SC volume fraction $\phi$ the $\mathrm{SC}$ transition, i.e. the supercurrent percolation, first appears along $z$, then along $y$, and only at much larger $\phi$ along the most conducting $x$-axis. Since $\phi$ increases with pressure $P$ (see Fig. 3 $\mathrm{d}$ inset), it explains the anisotropic SC transition observed in Refs. 15, 16, and 20, Notably, we do not need a questionable filamentary $z$-elongated shape of SC islands to describe these experiments: the effect emerges even for their

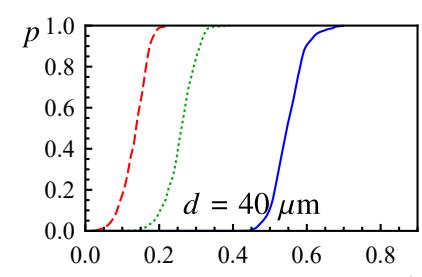

(a) $\phi$
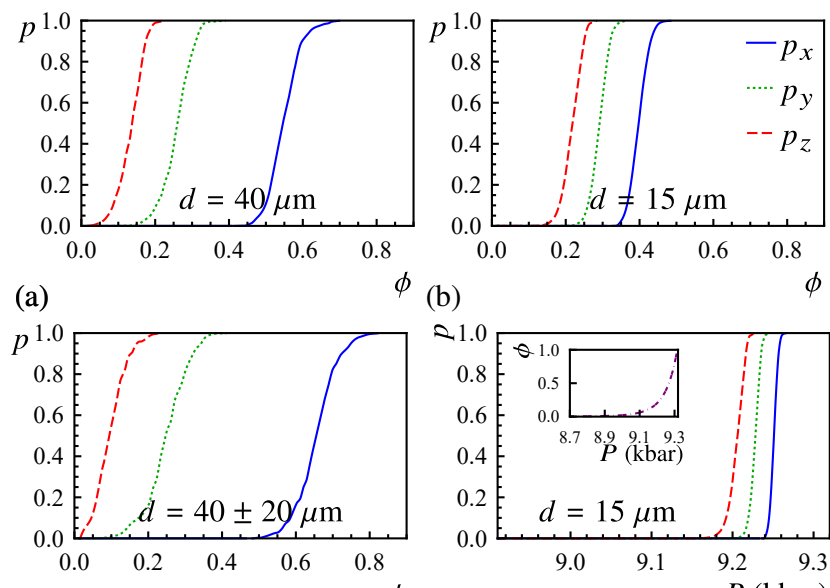

(c)

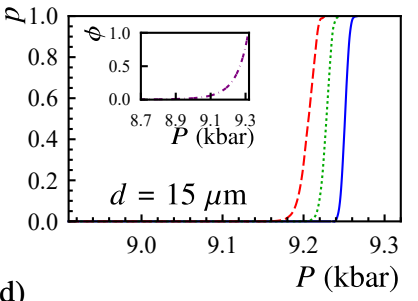

$\phi$ (d)

FIG. 3. Percolation probability $p$ along $x$ (solid blue), $y$ (dotted green) and $z$ (dashed red) axes as a function of SC volume fraction $\phi$. Spherical SC inclusions have diameter (a) $d=40 \mu \mathrm{m}$, (b) $d=15$ $\mu \mathrm{m}$, and (c) $d=40 \pm 20 \mu \mathrm{m}$ with standard deviation of $20 \mu \mathrm{m}$. (d) Dependence of $p$ on pressure $P$ along main axes for spherical SC inclusions of $d=15 \mu \mathrm{m}$, calculated from Fig. 3p using the experimental data $\phi(P)$ (inset) extracted from Tab. 1 of Ref. 14

opposite flattened shape. Thus, our scenario reconciles the relevant experimental facts on $\mathrm{SC}$ onset in $(\mathrm{TMTSF})_{2} \mathrm{PF}_{6}$ and (TMTSF) ${ }_{2} \mathrm{ClO}_{4}$ : (i) the anisotropy of SC onset [15, 16, 20, 39], (ii) the observation of AMRO [16, 39], and (iii) the strong $H_{c 2}$ enhancement in the DW/SC coexistence region [13, 20, 39].

Our numerical result of anisotropic percolation threshold can be easily understood. In thin elongated samples with $L_{x} \gg L_{z}$ the probability to find a chain of $n \approx L_{z} / d \sim 1$ connected SC islands, needed for percolation along the shortest edge of a sample, is much larger than to find a chain of length $N \approx L_{x} / d \gg 1$ for the percolation along the longest edge. This simple argument is illustrated in Fig. 4 .

Evidently, with the increase in sample length $L_{x}$ and thickness $L_{y}$ at other parameters $d, L_{z}, \phi$ fixed, the percolation probability $p_{z}$ along the sample thickness grows. At small $p_{z} \ll 1, p_{z} \propto L_{x} \times L_{y}$. The anisotropy of SC percolation transition also depends on the ratio of SC grain size $d$ and of the sample thickness $L_{z}$ (see Figs. 3). This dependence is important because it allows an experimental study of the typical size $d$ of SC islands in various materials and far from the sample boundary using resistivity measurements.

To investigate the main features of this dependence, we calculated percolation probabilities $p_{x}$ and $p_{y}$ as a function of diameter $d$ of SC islands in a 2D rectangular sample of dimension $L_{x} \times L_{y}$. The results are shown in Figs. $4 \mathrm{~b}$-e with $\phi_{i}^{c}$ plotted instead of $p_{i}$, where $i \in\{x, y\} . \phi_{i}^{c}$ is found by solving the equation $p_{i}^{c}(\phi)=1 / 2$. We found that $\phi_{x}^{c}$ depends weakly on $L_{x} / L_{y}$ (Figs. 4 4 ,d), but strongly on $L_{y} / d$ (Figs. 4b,e). It means that the percolation threshold along the sample length is more sensitive to the size of SC islands than to the sample length. Comparison of Figs. 3 $\mathrm{b}$ and $3 \mathrm{~b}$ shows a similar dependence of $p_{x}(\phi)$ on $d$ in 3D case. On the contrary, as $\phi_{y}^{c}$ depends strongly on $L_{x} / L_{y}$ (Figs. 4 c,d) and weakly on $L_{y} / d$ 


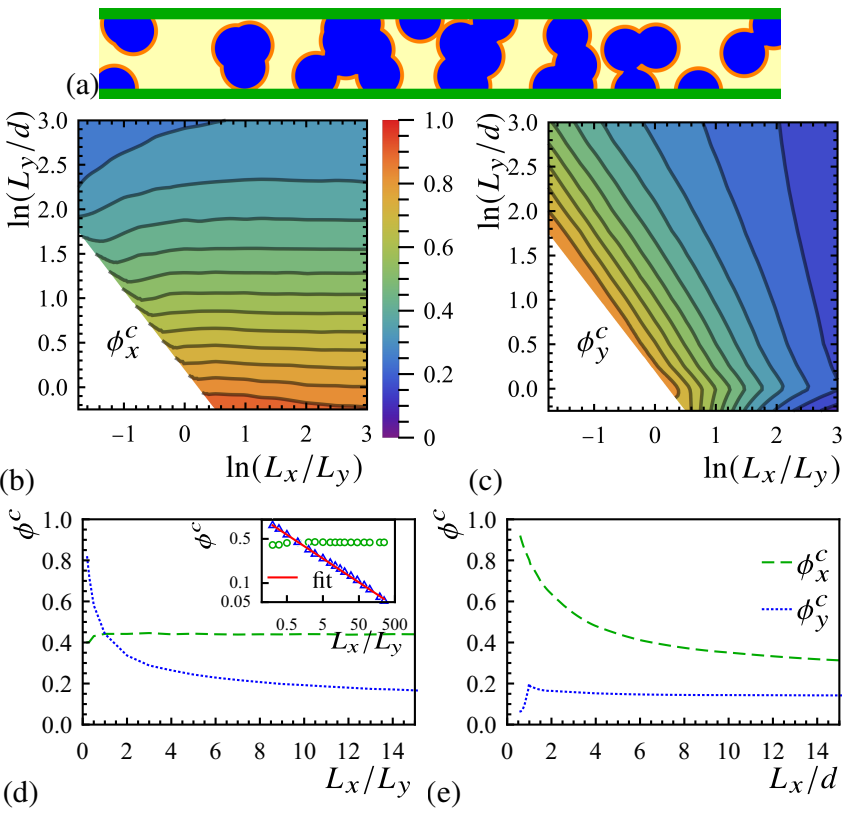

FIG. 4. Dependence of percolation threshold $\phi^{c}$ on sample shape and size in 2D. (a) Circular SC islands (blue) with diameter $d=$ 0.3 are randomly distributed inside a rectangular sample (yellow) of dimensions $10 \times 1$, forming SC channels between contact electrodes (green). It shows that the percolation along sample thickness is much easier than along sample length. $(\mathrm{b}, \mathrm{c}) \phi_{x}^{c}, \phi_{y}^{c}$ as a functions of sample length to sample thickness ratio $L_{x} / L_{y}$ and of sample thickness to $\mathrm{SC}$ island diameter ratio $L_{y} / d$. (d) Dependence of $\phi_{x}^{c}$ and $\phi_{y}^{c}$ on $L_{x} / L_{y}$ for $d=L_{y} / 5$. Same plot in logarithmic scale (inset) shows that $\phi_{y}^{c} \propto\left(L_{x} / L_{y}\right)^{-0.38}$. (e) Dependence of $\phi_{x}^{c}$ and $\phi_{y}^{c}$ on $L_{y} / d$ for $L_{x} / L_{y}=20$.

(Figs. 4k,e), percolation threshold along sample thickness is more sensitive to the length of the sample than to the size of SC islands. When $d \approx L_{y}$ we see a cusp in $\phi_{y}^{c}$, representing percolation due to a single SC inclusion. Numerical fitting shows $\phi_{y}^{c} \propto\left(L_{x} / L_{y}\right)^{-\alpha}$, where $\alpha$ increases with $d: \alpha \approx 0.34$ at $d=L_{y} / 7$ and $\alpha \approx 0.41$ at $d=L_{y} / 3$. Difference in $\phi_{x}^{c}-\phi_{y}^{c}$ grows with the increase in $L_{x} / L_{y}$ (Fig. 44) and $d$ (Fig. 4h). Similar effect is also observed in Figs. 3 3 ,b for 3D case. Hence, the anisotropy of SC onset grows when the sample becomes thinner and longer, and when the SC grain size $d$ increases. This shows the importance of finite-size effects for the anisotropy of SC onset.

\section{DISCUSSION AND SUMMARY}

Due to layered crystal structure of the high- $T_{c}$ and organic superconductors, usually, thin flat samples are produced during the crystal growth [40]. Temperature-dependent resistivity anisotropy is observed in most high- $T_{C}$ superconductors, both cuprate [41-48] and Fe-based [32, 49- 52]. According to our model, it depends on (i) the shape of SC islands, (ii) normalstate conductivity anisotropy, entering Eqs. (1.47), and (iii) the sample shape and size. The comparison of observed resistivity anisotropy of any sign with our model gives information about typical size and shape of SC islands deep inside the sample. An anisotropic resistivity drop above $T_{c}$ and the applicability of Eqs. (1 4) do not require finite-size effects and persist at $d \ll L$ and arbitrary sample shape. However, for anisotropic zero-resistance $T_{c}$ the finite sample size $L \lesssim 10^{3} d$ in any direction is important. The doping-formed inhomogeneities in cuprates are, usually, $\sim 10 \mathrm{~nm} \ll L_{z} \gtrsim 1 \mu \mathrm{m}$. Nevertheless, they may form much larger clusters, even $\gtrsim 1 \mu \mathrm{m}$ as in $\mathrm{HgBa}_{2} \mathrm{CuO}_{4+y}$ (see Figs. 1g or 3d of Ref. [53]). Interplay between SC and DW also leads to larger SC domains. Tiny and flat samples, required for anisotropic zero-resistance $T_{c}$, can be made artificially. In FeSe this led to an amazing observation [32, 52]: in $z$-direction $T_{c}$ increases from $8 \mathrm{~K}$ to $12 \mathrm{~K}$ as the sample thickness decreases from $300 \mathrm{~nm}$ to $\sim 50 \mathrm{~nm}$, while along the $a-b$ plane $T_{c} \approx 8 \mathrm{~K}$ remains unchanged. Our model explains this effect also.

Superconductivity onsets heterogeneously in all known high-temperature superconductors, as confirmed by numerous scanning tunneling microscopy and spectroscopy measurements [53-60]. However, these and other elaborated experimental techniques provide detailed information about the electronic structure at the surface, which may differ from the structure deep in the bulk. The proposed effect allows one to estimate the typical size and shape of SC islands far from the surface by measuring the temperature dependence of resistivity along three main axes in the samples or artificial bridges of thickness comparable to or 1-3 orders less than the expected size of SC grains. This knowledge is helpful for understanding the properties and electronic structure across the phase diagram of various high- $T_{c}$ superconductors.

To summarize, we provide an explanation for the puzzling experimental data in organic superconductors, where the SC onset is highly anisotropic. This explanation is based on heterogeneous SC nucleation. Above $T_{c}$ the resistance decreases anisotropically because the rare SC islands reduce resistivity most strongly along the least conducting direction, as described analytically in Sec. III A using the effective-medium approximation, applicable to samples of any large size and small fraction of SC phase. To explain the anisotropic zeroresistance onset or $T_{c}$, in Sec. IIB we performed the numerical calculation of current percolation probability via SC islands in finite-size samples of various shape. This calculation revealed a remarkable anisotropy of the percolation threshold, which depends strongly on the relative size and shape of samples and $\mathrm{SC}$ islands. The proposed effect allows one to estimate the typical size and shape of SC grains far from the sample boundary using resistivity measurements. This effect is rather general for inhomogeneous superconductors and most pronounced when the non-SC material has anisotropic resistivity, as cuprate and Fe-based superconductors, and/or when the sample shape is anisotropic.

\section{ACKNOWLEDGMENTS}

This article is partly supported by the Ministry of Science and Higher Education of the Russian Federation in the framework of Increase Competitiveness Program of MISiS, by 
RFBR grant \# 21-52-12027, and by the "Basis" Foundation for development of theoretical physics and mathematics. V. D. K. acknowledges the MISiS project \# K2-2020-001, and K. K. K. the MISiS support project for young research engineers and RFBR \# 19-32-90241 \& 19-31-27001. P. D. G. acknowledges the State Assignment \# 0033-2019-0001 and RFBR grants \# 19-02-01000 \& 21-52-12043.

\section{Appendix A: Derivation of resistivity of heterogeneous anisotropic compound in the self-consistent approximation}

\section{Isotropic case with ellipsoidal inclusions}

In the self-consistent approximation (SCA), the effect of all the material outside any inclusion is to produce a homogeneous medium whose effective conductivity $\sigma_{i i}^{*}$ is the unknown to be calculated [35]. The diagonal components of the effective conductivity tensor $\sigma_{i i}^{*}$ along the axis $i \in\{x, y, z\}$ of a heterogeneous media with unidirectionally aligned isotropic ellipsoidal inclusions in SCA can be calculated from Eqs. (18.18) and (18.19) of Ref. 35:

$$
\sum_{j}^{N} \frac{\phi^{j}\left(\sigma^{j}-\sigma_{i i}^{*}\right) \sigma_{i i}^{*}}{\sigma_{i i}^{*}+A_{i}^{*}\left(\sigma^{j}-\sigma_{i i}^{*}\right)}=0,
$$

where $j$ numerates the phase, $\phi^{j}$ is its volume fraction, $\sigma^{j}$ is its conductivity, which is assumed to be isotropic, and the diagonal components $A_{i}^{*}$ of depolarization tensor for ellipsoidal inclusions with semiaxes $a_{i}^{*}$ are given by Eq. (3). In the next subsection we generalize these results for the case of anisotropic conductivities $\sigma_{i i}^{j}$ of constituent phases. For only two different phases, $m$ and $s$, with isotropic conductivities $\sigma^{m}$ and $\sigma^{s}$, according to Eq. (A1), the effective conductivity $\sigma_{i i}^{*}$ along the axis $i$ of such a heterogeneous media satisfies the equation:

$$
\frac{(1-\phi)\left(\sigma^{m}-\sigma_{i i}^{*}\right) \sigma_{i i}^{*}}{\sigma_{i i}^{*}+A_{i}^{*}\left(\sigma^{m}-\sigma_{i i}^{*}\right)}+\frac{\phi\left(\sigma^{s}-\sigma_{i i}^{*}\right) \sigma_{i i}^{*}}{\sigma_{i i}^{*}+A_{i}^{*}\left(\sigma^{s}-\sigma_{i i}^{*}\right)}=0,
$$

where $\phi$ is the volume fraction of phase $s$, which in our case is superconducting (SC). The conductivity of SC inclusions $\sigma^{s} \rightarrow \infty$. Then from Eq. (A2) we obtain a simple formula for the effective conductivity:

$$
\sigma_{i i}^{*}=\frac{\sigma^{m} A_{i}^{*}}{A_{i}^{*}-\phi} .
$$

\section{Anisotropic case with ellipsoidal inclusions}

The generalization of Eq. (A3) to the case of anisotropic conductivity $\sigma^{m}$ of the parent media is performed by the mapping of the initial anisotropic problem to an isotropic one in a similar way as used in Ref. 36 for the derivation of effective conductivity in the Maxwell-Garnett approximation (MGA), given by Eqs. (1) and (3). Let $\boldsymbol{J}$ and $V$ be the current density and the electric potential respectively in the real space, and $\sigma_{i i}^{m}$ be the conductivity components of the parent phase. The electrostatic continuity equation in real space is written as:

$$
-\nabla \cdot \boldsymbol{J}=\sum_{i} \frac{\partial}{\partial r_{i}}\left(\sigma_{i i}^{m} \frac{\partial V}{\partial r_{i}}\right)=0,
$$

where $i \in\{x, y, z\}$. After the mapping, i.e. the change of coordinates $r_{i}$ as:

$$
r_{i}=r_{i}^{*} \sqrt{\eta_{i}}, \eta_{i}=\sigma_{i i}^{m} / \sigma_{x x}^{m},
$$

with the simultaneous change of conductivity to $\sigma^{m}=\sigma_{x x}^{m}$, Eq. (A4) transforms to the electrostatic continuity equation for an isotropic media:

$$
-\nabla \cdot \boldsymbol{J}=\sum_{i} \frac{\partial}{\partial r_{i}^{*}}\left(\sigma^{m} \frac{\partial V}{\partial r_{i}^{*}}\right)=0 .
$$

Coordinate dependence of the electrostatic potential $V(x, y, z)$ in an inhomogeneous medium, given by solutions of the equations A4 or A6 with proper boundary conditions, determines the effective conductivity of this inhomogeneous medium. Consequently, the initial problem of conductivity in anisotropic media with some boundary conditions can be mapped to the conductivity problem in isotropic media with new boundary conditions, obtained from the initial boundary conditions by anisotropic dilatation given in Eq. A5. These boundary conditions are determined both by the sample boundaries and by the inclusions of second phase. If these inclusions have ellipsoidal shape with the principal semiaxes $a_{i}$, then after the mapping to the isotropic media these inclusions keep an ellipsoidal shape but change the principal semiaxes to:

$$
a_{i}^{*}=a_{i} / \sqrt{\eta_{i}} .
$$

Eqs. (A3) and (3) with semiaxes $a_{i}^{*}$ give the effective conductivity in the mapped space. Making the reverse mapping to the real space, we obtain the effective conductivity of initial heterogeneous media in real space in SCA:

$$
\sigma_{i i}=\frac{\sigma_{i i}^{m} A_{i}^{*}}{A_{i}^{*}-\phi},
$$

which gives Eq. (2).

Note that in the final formula $(\mathrm{A} 8)$ the effective conductivity $\sigma_{i i}$ in the real space depends on the parameters $A_{i}^{*}$ and $a_{i}^{*}$ in the mapped space. This is because the coordinate dependence of electrostatic potential $V\left(r_{i}\right)$ in the real space is obtained from the electrostatic potential $V^{*}\left(r_{i}^{*}\right)$ in the mapped space (with semiaxes $a_{i}^{*}$ ) via the simple substitution of Eq. A5): $V\left(r_{i}\right)=$ $V^{*}\left(r_{i}^{*}\right)$. The dilatation $r_{i} \rightarrow r_{i} / \sqrt{\eta_{i}}$ changes $\sqrt{\eta_{i}}$ times the electric field $E_{i}\left(r_{i}\right)=-\nabla_{i} V\left(r_{i}\right)$, while the electric current $J_{i}=\sigma_{i i} E_{i}$ changes $1 / \sqrt{\eta_{i}}$ times, because the local conductivity $\sigma_{i i}^{m}$ changes $1 / \eta_{i}$ times. The effective conductivity $\sigma_{i i}$ also changes $1 / \eta_{i}$ times: $\sigma_{i i}=J_{i} \bar{E}_{i}$, where the averaged (over sample size $\left.L_{i}\right)$ electric field $\bar{E}_{i}=L_{i} /\left[V\left(r_{i}=0\right)-V\left(r_{i}=L_{i}\right)\right]$ changes $1 / \sqrt{\eta_{i}}$ times due to the dilatation. 


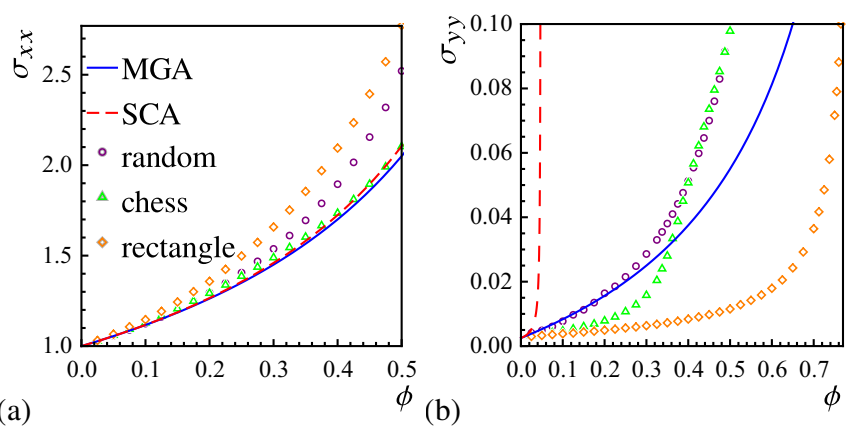

FIG. 5. The conductivity of an anisotropic (square $1 \times 1$ ) heterogeneous media with superconducting inclusions calculated using analytical models, Eq. (1) for MGA and Eq. (2) or A8 for SCA, and numerically for three different distributions of SC islands: random, rectangular and chess order. In SCA $\sigma_{y y}(\phi)$ goes up sharply and even diverges at $\phi \sim \eta \ll 1$, which drastically contradicts the numerical results.

\section{Appendix B: Comparison of the results of bulk analytical models and numerical calculations}

In this section we compare the results, given by analytical formulas (1)-(3) obtained in the Maxwell-Garnett (MGA) and self-consistent (SCA) approximations, with the numerical calculations in 2D case (see Fig. 5). This allows to estimate the applicability of these two bulk analytical models to describe real experiments on conductivity in heterogeneous superconductors. The calculated conductivity along two axes, $x$ and $y$, for a square heterogeneous media of conductivity $\sigma_{x x}^{0}=1$ and $\sigma_{y y}^{0}=\eta=1 / 400$ with circular superconducting islands as a function of their volume fraction $\phi$ is shown in Fig. 5 . For numerical calculations three different distributions of SC islands are considered: random, rectangular and chess order. For rectangle order our numerical calculations give the largest conductivity $\sigma_{x x}(\phi)$ along the most conducting axis and the smallest conductivity $\sigma_{y y}(\phi)$ along the most conducting direction. For conductivity $\sigma_{x x}(\phi)$ all approximations, both numerical and analytical, give similar results (see Fig. 5a). However, $\sigma_{y y}(\phi)$ in various approximations differ much, as shown in Fig. 5p. The numerical calculations of $\sigma_{y y}(\phi)$ for all three distributions of SC islands give rather close results, but the analytical models MGA and SCA differ very strongly. The MGA approximation for $\sigma_{y y}(\phi)$ is much closer to the numerical results than SCA: the conductivity $\sigma_{y y}$ in SCA deviates crucially and diverges at $\phi \sim \eta \ll 1$. This calculation illustrates the known fact [35] that SCA, usually, gives qualitatively incorrect results in the limit of strong contrast between the conductivities of two phases in heterogeneous media, es- pecially in the limit of strong anisotropy.

\section{Appendix C: Details of fits and calculations}

In plotting Fig. $2 \mathrm{~d}$ we assume that the magnetic field $B_{z}=0.22 \mathrm{~T}$ is strong enough to suppress superconductivity. In fact, such a field at $P=8.3$ kbar reduces the SC transition temperature from $T_{c}\left(B_{z}=0\right) \approx 1.1 \mathrm{~K}$ to $T_{c}\left(B_{z}=0.22 \mathrm{~T}\right) \approx 0.3 \mathrm{~K}$. Hence, these data can be used to determine $\phi(T>0.3 \mathrm{~K})$. The magnetic field $B_{z}=0.22 \mathrm{~T}$ also leads to small metallic magnetoresistance $\rho_{b}(B)$, which is almost temperature independent at $T_{c}<T<1.5 \mathrm{~K}$ (see Fig. 4b of Ref. 15). Therefore we take it into account in our calculation of $\phi(T)$ by the offset $\rho_{y}^{0}(T)=\rho_{y}\left(T, B_{z}=0.22 \mathrm{~T}\right)-\left[\rho_{y}(T=\right.$ $\left.\left.1.15 \mathrm{~K}, B_{z}=0.22 \mathrm{~T}\right)-\rho_{y}\left(T=1.15 \mathrm{~K}, B_{z}=0\right)\right]$.

The percolation probability in Figs. 3, 4 was calculated numerically using Monte-Carlo simulation. For each distribution of diameters $d=\mu \pm \sigma$, which is taken Gaussian with a half-width $\sigma$, a random state with proper number of spherical inclusions in a box with given dimensions $\left(L_{x} \times L_{y} \times L_{z}=3 \times 0.2 \times 0.1 \mathrm{~mm}^{3}\right.$ in Fig. 3 and various $L_{x} \times L_{y}$ in Fig. 4) was generated. The number of SC inclusions is determined by the fixed volume fraction $\phi$ of SC phase. Each state is associated with a graph whose vertices are $\mathrm{SC}$ islands. The vertices of the graph are connected by edges if the corresponding inclusions overlap. Thus, the problem of detecting the presence of percolation is reduced to finding the connected components of the graph, which contain the vertices corresponding to SC inclusions on the opposite sample edges. For each state along each axis the percolation, i.e. the existence of a continuous path via intersecting inclusions, was checked, and the averaging over random realizations was made. Depending on the parameters, from $10^{4}$ to $10^{5}$ generated realizations were enough to estimate the average probability of percolation in our calculations.

The conductivity of an anisotropic media (in Fig. 5p was calculated numerically by solving the electrostatic continuity equation (A4) for the heterogeneous medium using the finite element method.

A quantitative comparison with experiment requires the exact functions $\phi(P)$ and $\phi(T)$, which are known only approximately. Fig. 2d, based on resistivity in MGA, overestimates $\phi(T)$, because MGA gives a lower bound of conductivity in heterogeneous media [35]. On contrary, Fig. 3c inset, based on the resistivity fit above $T_{c}$ in the metal/SDW phase [14], underestimates $\phi(P)$, because the volume fraction of SC phase at $T<T_{c}$ should be larger than the volume fraction of metal phase at $T_{c}<T \ll T_{C S D W}$ for two reasons: (i) superconducting phase has lower energy than metallic phase, and (ii) the SC proximity effect increases the effective SC volume fraction.
[1] A. M. Gabovich, A. I. Voitenko, J. F. Annett, and M. Ausloos, Supercond. Sci. Technol. 14, R1 (2001)

[2] A. M. Gabovich, A. I. Voitenko, and M. Ausloos, Phys. Rep. 367, 583 (2002)
[3] P. Monceau, Adv. Phys. 61, 325 (2012).

[4] J. Chang, E. Blackburn, A. Holmes, N. Christensen, J. Larsen, J. Mesot, R. Liang, D. Bonn, W. Hardy, A. Watenphul, M. Zimmermann, E. Forgan, and S. Hayden, Nature Phys 8, 871 (2012) 
[5] S. Blanco-Canosa, A. Frano, T. Loew, Y. Lu, J. Porras, G. Ghiringhelli, M. Minola, C. Mazzoli, L. Braicovich, E. Schierle, E. Weschke, M. Le Tacon, and B. Keimer, Phys. Rev. Lett. 110, 187001 (2013)

[6] W. Tabis, B. Yu, I. Bialo, M. Bluschke, T. Kolodziej, A. Kozlowski, E. Blackburn, K. Sen, E. Forgan, M. Zimmermann, Y. Tang, E. Weschke, B. Vignolle, M. Hepting, H. Gretarsson, R. Sutarto, F. He, M. Le Tacon, N. Barišić, G. Yu, and M. Greven, Phys. Rev. B 96, 134510 (2017)

[7] Q. Si, R. Yu, and E. Abrahams, Nat Rev Mater 1, 16017 (2016)

[8] X. Liu, L. Zhao, S. He, J. He, D. Liu, D. Mou, B. Shen, Y. Hu, J. Huang, and X. J. Zhou, J. Phys.: Condens. Matter 27, 183201 (2015)

[9] C.-S. Lian, C. Si, and W. Duan, Nano Lett. 18, 2924 (2018)

[10] K. Cho, M. Kończykowski, S. Teknowijoyo, M. Tanatar, J. Guss, P. Gartin, J. Wilde, A. Kreyssig, R. McQueeney, A. Goldman, V. Mishra, P. Hirschfeld, and R. Prozorov, Nat Commun 9, 2796 (2018)

[11] T. Ishiguro, K. Yamaji, and G. Saito, Organic Superconductors (Springer Berlin Heidelberg, 1998).

[12] A. Lebed, ed., The Physics of Organic Superconductors and Conductors (Springer Berlin Heidelberg, 2008).

[13] I. J. Lee, P. M. Chaikin, and M. J. Naughton, Phys. Rev. Lett. 88, 207002 (2002).

[14] T. Vuletić, P. Auban-Senzier, C. Pasquier, S. Tomić, D. Jérome, M. Héritier, and K. Bechgaard, Eur. Phys. J. B 25, 319 (2002)

[15] N. Kang, B. Salameh, P. Auban-Senzier, D. Jerome, C. R. Pasquier, and S. Brazovskii, Phys. Rev. B 81, 100509(R) (2010)

[16] A. Narayanan, A. Kiswandhi, D. Graf, J. Brooks, and P. Chaikin, Phys. Rev. Lett. 112, 146402 (2014)

[17] I. J. Lee, S. E. Brown, W. Yu, M. J. Naughton, and P. M. Chaikin, Phys. Rev. Lett. 94, 197001 (2005).

[18] I. J. Lee, M. J. Naughton, G. M. Danner, and P. M. Chaikin, Phys. Rev. Lett. 78, 3555 (1997)

[19] I. J. Lee, S. E. Brown, W. G. Clark, M. J. Strouse, M. J. Naughton, W. Kang, and P. M. Chaikin, Phys. Rev. Lett. 88, 017004 (2001)

[20] Y. A. Gerasimenko, S. V. Sanduleanu, V. A. Prudkoglyad, A. V. Kornilov, J. Yamada, J. S. Qualls, and V. M. Pudalov, Phys. Rev. B 89, 054518 (2014)

[21] S. Yonezawa, C. A. Marrache-Kikuchi, K. Bechgaard, and D. Jerome, Phys. Rev. B 97, 014521 (2018)

[22] D. Andres, M. V. Kartsovnik, W. Biberacher, K. Neumaier, E. Schuberth, and H. Muller, Phys. Rev. B 72, 174513 (2005)

[23] P. D. Grigoriev, Phys. Rev. B 77, 224508 (2008)

[24] S. Brazovskii and N. Kirova, Sov. Sci. Rev. A 5, 99 (1984).

[25] W. P. Su, S. Kivelson, and J. R. Schrieffer, in Physics in One Dimension, Springer Series in Solid-State Sciences, edited by J. Bernascony and T. Schneider (Springer Berlin Heidelberg, 1981) pp. 201-211.

[26] P. D. Grigoriev, Physica B 404, 513 (2009).

[27] L. P. Gor'kov and P. D. Grigoriev, Europhys. Lett. 71, 425 (2005)

[28] L. P. Gor'kov and P. D. Grigoriev, Phys. Rev. B 75, 020507(R) (2007)

[29] The critical pressure $P_{c}$, where SDW disappears, measured by two different groups has different values: $\left.P_{c} \approx 6.4 \mathrm{kbar}[13] 16\right]$ or $P_{c} \approx 9.5 \mathrm{kbar}[14,15]$. This disagreement is related to different experimental technique of measuring pressure rather than to different samples. It does not affect the microscopic structure of coexistence phase, because other measured properties of (TMTSF) ${ }_{2} \mathrm{PF}_{6}$ coincide except this pressure offset [14-16]. Similar pressure offsets apply to other pressure values $P_{c 0}, P_{c 1}$, $P_{c 2}$ on the phase diagram in Fig. 1 .
[30] M. Tinkham, Introduction to superconductivity, 2nd ed., International series in pure and applied physics (McGraw-Hill, Inc., New York, 1996).

[31] A. L. Efros, Physics and Geometry of Disorder: Percolation Theory, Science for Everyone (Imported Pubn, 1987).

[32] A. A. Sinchenko, P. D. Grigoriev, A. P. Orlov, A. V. Frolov, A. Shakin, D. A. Chareev, O. S. Volkova, and A. N. Vasiliev, Phys. Rev. B 95, 165120 (2017).

[33] P. D. Grigoriev, A. A. Sinchenko, K. K. Kesharpu, A. Shakin, T. I. Mogilyuk, A. P. Orlov, A. V. Frolov, D. S. Lyubshin, D. A. Chareev, O. S. Volkova, and A. N. Vasiliev, JETP Lett. 105, 786 (2017)

[34] Here and below the upper index " 0 " denotes the parent non-SC compound.

[35] S. Torquato, Random Heterogeneous Materials (Springer New York, 2002).

[36] S. S. Seidov, K. K. Kesharpu, P. I. Karpov, and P. D. Grigoriev, Phys. Rev. B 98, 014515 (2018)

[37] H. Schwenk, K. Andres, F. Wudl, and E. Aharon-Shalom, Solid State Commun. 45, 767 (1983)

[38] S. V. Dordevic, D. N. Basov, and C. C. Homes, Sci Rep 3, 1713 (2013)

[39] Y. A. Gerasimenko, V. A. Prudkoglyad, A. V. Kornilov, S. V. Sanduleanu, J. S. Qualls, and V. M. Pudalov, JETP Lett. 97, 419 (2013)

[40] J. Karpinski, G. I. Meijer, H. Schwer, R. Molinski, E. Kopnin, K. Conder, M. Angst, J. Jun, S. Kazakov, A. Wisniewski, R. Puzniak, J. Hofer, V. Alyoshin, and A. Sin, Supercond. Sci. Technol 12, R153 (1999).

[41] J.-S. Zhou, J. B. Goodenough, B. Dabrowski, and K. Rogacki, Phys. Rev. Lett. 77, 4253 (1996).

[42] N. E. Hussey, K. Nozawa, H. Takagi, S. Adachi, and K. Tanabe, Phys. Rev. B 56, R11423 (1997)

[43] S. Martin, A. T. Fiory, R. M. Fleming, L. F. Schneemeyer, and J. V. Waszczak, Phys. Rev. Lett. 60, 2194 (1988)

[44] S. Ono and Y. Ando, Phys. Rev. B 67, 104512 (2003)

[45] J. Garcia-Barriocanal, A. Kobrinskii, X. Leng, J. Kinney, B. Yang, S. Snyder, and A. M. Goldman, Phys. Rev. B 87, $024509(2013)$

[46] Y. Ando, K. Segawa, S. Komiya, and A. N. Lavrov, Phys. Rev. Lett. 88, 137005 (2002)

[47] V. N. Zverev, D. V. Shovkun, and I. G. Naumenko, JETP Lett. 68, 332 (1998)

[48] V. N. Zverev and D. V. Shovkun, JETP Lett. 72, 73 (2000)

[49] M. A. Tanatar, N. Ni, A. Thaler, S. L. Bud'ko, P. C. Canfield, and R. Prozorov, Phys. Rev. B 82, 134528 (2010)

[50] M. A. Tanatar, E. C. Blomberg, A. Kreyssig, M. G. Kim, N. Ni, A. Thaler, S. L. Bud'ko, P. C. Canfield, A. I. Goldman, I. I. Mazin, and R. Prozorov, Phys. Rev. B 81, 184508 (2010)

[51] V. N. Zverev, A. V. Korobenko, G. L. Sun, D. L. Sun, C. T. Lin, and A. V. Boris, JETP Lett. 90, 130 (2009)

[52] T. I. Mogilyuk, P. D. Grigoriev, K. K. Kesharpu, I. A. Kolesnikov, A. A. Sinchenko, A. V. Frolov, and A. P. Orlov, Phys. Solid State 61, 1549 (2019)

[53] G. Campi, A. Bianconi, N. Poccia, G. Bianconi, L. Barba, G. Arrighetti, D. Innocenti, J. Karpinski, N. D. Zhigadlo, S. M. Kazakov, M. Burghammer, M. v. Zimmermann, M. Sprung, and A. Ricci, Nature 525, 359 (2015).

[54] V. Kresin, Y. Ovchinnikov, and S. Wolf, Phys. Rep. 431, 231 (2006)

[55] K. M. Lang, V. Madhavan, J. E. Hoffman, E. W. Hudson, H. Eisaki, S. Uchida, and J. C. Davis, Nature 415, 412 (2002).

[56] W. D. Wise, K. Chatterjee, M. C. Boyer, T. Kondo, T. Takeuchi, H. Ikuta, Z. Xu, J. Wen, G. D. Gu, Y. Wang, and E. W. Hudson, 
Nature Phys 5, 213 (2009)

[57] A. Kamlapure, T. Das, S. C. Ganguli, J. B. Parmar, S. Bhattacharyya, and P. Raychaudhuri, Sci Rep 3, 2979 (2013)

[58] K. Gofryk, M. Pan, C. Cantoni, B. Saparov, J. E. Mitchell, and A. S. Sefat, Phys. Rev. Lett. 112, 047005 (2014)

[59] Y. G. Naidyuk, G. Fuchs, D. A. Chareev, and A. N. Vasiliev,
Phys. Rev. B 93, 144515 (2016).

[60] A. Fente, A. Correa-Orellana, A. E. Bohmer, A. Kreyssig, S. Ran, S. L. Budko, P. C. Canfield, F. J. Mompean, M. GarcíaHernández, C. Munuera, I. Guillamón, and H. Suderow, Phys. Rev. B 97, 014505 (2018) 